\title{
New PIC Scheme for Multi-Carrier DS-CDMA
}

\author{
Guo-Xiong Xu \\ School of Electric and Information Engineer, Zhongyuan University of Technology, Zhengzhou, China \\ E-mail: xgxh88@sina.com
}

\begin{abstract}
This paper explores a new parallel interference cancellation (PIC) scheme for multi-carrier DS-CDMA. For the PIC scheme, frequency diversity technique and the linearization of soft decision function are used to simplify the complexity and improve the system performance. The theoretical analysis over the proposed PIC scheme is detailed, numerical simulations are performed, and main conclusions are drawn as: (1) for the proposed PIC scheme, whether over Rayleigh fading channel or over additive white Gaussian moise (AWGN) channel, more interference cancellation (IC) stages can bring larger performance gain, but with the further increase in the number of IC stages, the performance improves less dominantly; (2) in case of the same IC stages, for the proposed PIC scheme, the linearization of soft decision function will bring the reduction of computation complexity with an ignorable performance loss.
\end{abstract}

Keywords- multi-carrier; DS-CDMA; parallel interference cancellation; multi-access interference

\section{INTRODUCTION}

Multi-carrier CDMA, which is a combination of both multi-carrier modulation (MCM) and code-division multiple access (CDMA), has superior capabilities of suppressing multi-access interference (MAI) among active users and multi-path fading [1]. For multi-carrier CDMA systems, data sequence is performed first spectrum-spreading and subsequent multi-carrier modulation. In general, multicarrier CDMA has two types: OFDM-CDMA and MC-DSCDMA. The main difference between both types is that the spectrum-spreading of the former is based on frequency domain and that of the latter is based on time domain. Both types of multi-carrier CDMA were explored in detail in [1] and[2], respectively. Two types of multi-carrier CDMA systems have a similar capability of combating multi-access interference and narrow-band interferenc, and therefore exhibit a dominant performance advantage, compared to conventional single-carrier DS-CDMA.

Like single-carrier CDMA, multi-carrier CDMA is suffered from multi-access interference (MAI), which is as a result of non-orthogonality between usres' signiture sequences. To a great degree, it is due to MAI that the performance degradation of multi-carrier CDMA occurs. In order to improve the performance of multi-carrier CDMA, it is necessary to adopt effective stategies to suppress or cancel MAI. Among these stategies is multi-user detection (MUD) [3-4]. Multi-user detection schemes are generally sorted as linear MUD and nonlinear MUD. The former includes MMSE detector and decorrelation detector, while the latter is divided into successive interference cancellation (SIC) and parallel interference cancellation (PIC). L.Fang et al analyzed successive interference cancellation (SIC) for multi-carrier DS-CDMA [5]. The related results show that SIC can effectively combat MAI and improve the performance for multi-carrier DS-CDMA systems. PIC for multi-carrier DS-CDMA was also discussed in detail in [6], which shows that PIC can improve the system performance dominantly.

In this paper, a new parallel interference cancellation scheme is proposed for multi-carrier DS-CDMA. For the proposed scheme, frequentcy diversity combining (FDC) technology is considered and soft decision function is derived and linearized, respectively. Detailed analysis and discussion are made for the proposed PIC scheme of multicarrier DS-CDMA.

\section{DESCRIPTION OF THE PROPOSED SCHEME}

In this paper, we consider MC DS-CDMA model which was proposed and deeply discussed in [1]. Let $\mathrm{K}$ users' signals be transmitted. Each user's signal is firstly multiplied by distinct spreading sequences to perform frequencyspreading, and the corresponding spread signal is then replicated into multiple branches, each of which is modulated by designated sub-carrier.

Consider the effect of Rayleigh fading and additive white Gaussian noise (AWGN) on the signal transmission of multicarrier CDMA systems. Then the received signal can be expressed as

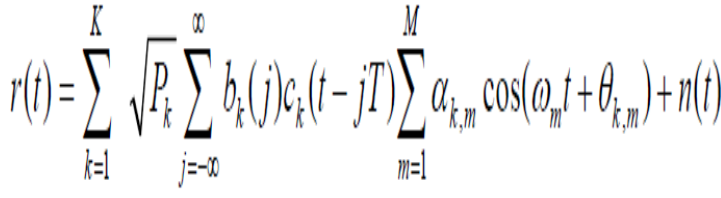

where $P_{k}$ is the k-th user's transmitted power, $b_{k}(j)$ is the $\mathrm{j}$-th transmitted symbol of the k-th user, $\alpha \mathrm{k}, \mathrm{m}$ is the fading factor which satifies Rayleigh distribution, $\omega \mathrm{m}$ is the $\mathrm{m}$-th sub-carrier frequency, $\mathrm{M}$ is the sub-carrier number, $n(t)$ is AWGN, and $c_{k}(t)=\sum_{n=0}^{N-1} c_{k}^{(n)} h\left(t-n T_{c}\right)$, where $\left\{c_{k}^{(n)}\right\}$ is the signiture sequence of the $\mathrm{k}$-th user, $\mathrm{N}$ is the spreading gain, and Tc is the chip duration.

The proposed PIC scheme for multi-carrier DS-CDMA, which is called MC-PIC scheme in the following, consists of multiple interference cancellation (IC) stages, each of which functions the same. With no loss of generality, we only give the description and analysis of the q-th IC stage, as shown in Figure 1. 


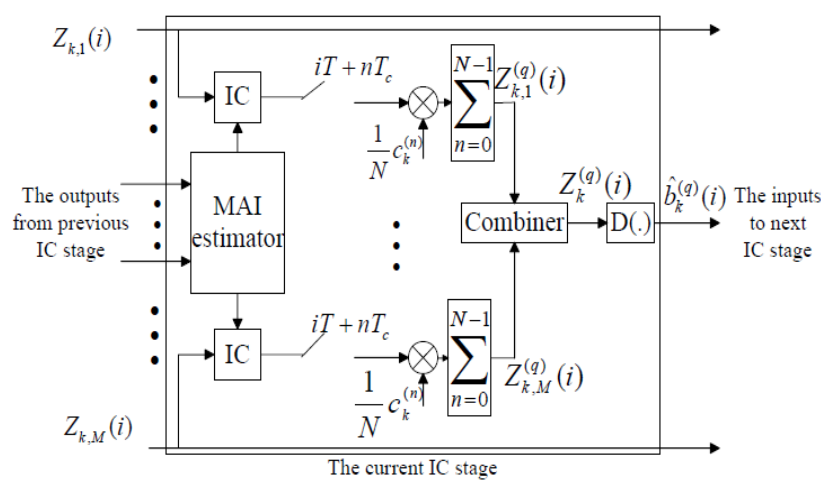

Figure 1. The $q$-th IC stage for the proposed MC-PIC scheme.

Consider MC-PIC scheme with Q IC stages. The receiver firstly makes despreading over the received signal $r(t)$, which is then sub-carrier demodulated and low-pass filtered to obtain match-filtered (MF) output of each sub-carrier for each user, and then the MF outputs are sent to multi-stage interference cancellor. After a number of stages of IC and frequency diversity combining, the outputs are used to make decisions to obtain the final results.

In the following, we mainly describe two parts of MCPIC receiver: (1) MF output, and (2) multi-stage IC.

\section{A MF Output}

The received signal $r(t)$ is processed by MF to obtain the correlation output, which is expressed as

$$
\begin{aligned}
& Z_{k, m}(i)=\frac{1}{N} \sum_{n=0}^{N-1} c_{k}^{(i N+n)}\left[\sqrt{E_{c}} \sum_{k^{\prime}=1}^{K} \sum_{j=-\infty}^{\infty} \alpha_{k^{\prime}, m} b_{k^{\prime}}(j) \sum_{n=0}^{N-1} c_{k^{\prime}}^{(j N+n)} x\left(t-j T-n T_{c}\right)+n_{k, m}(t)\right]= \\
& \sqrt{E_{c}} \alpha_{k, m} b_{k}(i)+I_{k, m}(i)+n_{k, m}(i)
\end{aligned}
$$

for the $\mathrm{m}$-th sub-carrier of the $\mathrm{k}$-th user, where $I_{k, m}(i)=\sum_{k^{\prime}=1, k^{\prime} \neq k}^{K} \frac{\sqrt{E_{c}}}{N} b_{k^{\prime}}(i) \rho_{k^{\prime}, k}$ is the interference from all other users's signals to user k, i.e., multi-access interference (MAI), $n_{k, m}(t)=\operatorname{LPF}\left(\operatorname{BPF}(n(t)) \sqrt{2} \cos \left(\omega_{m} t+\theta_{k, m}\right)\right) \quad, \quad \operatorname{LPF} \quad($.$) \quad and$ $\mathrm{BPF}($.)denote low-pass and band-pass filtering, respectively, $n_{k, m}(i)=\frac{1}{N} \sum_{n=0}^{N-1} c_{k}^{(i N+n)} n_{k, m}\left(i T+n T_{c}+\tau_{k}\right), \mathrm{x}(\mathrm{t})$ is the result due to inverse Fourier transform of $|H(f)|^{2}$, and $\mathrm{H}(\mathrm{f})$ is Fourier transform of $h(t)$.

It is clear that the first term $\sqrt{E_{c}} \alpha_{k, m} b_{k}(i)$ and the second term Ik,m(i) in (2) are, respectively, the desired signal component, and MAI component. The latter is expected to be suppressed to improve system performance.

The $Z_{k, m}(i)$ in (2) is subsequently input to the combiner for combining, and thus

$$
Z_{k}(i)=\sum_{m=1}^{M} w_{k, m} Z_{k, m}(i)
$$

where $w_{k, m}$ is combining gain.

Use $\mathrm{D}(\cdot)$ as decision function, and then we have symbol tentative decision as

$$
\hat{b}_{k}^{(0)}(i)=\mathrm{D}\left(Z_{k}(i)\right)
$$

The results by (4) are sent to multi-stage IC as intial inputs and updated at each IC stage.

\section{B The q-th IC Stage}

In this IC stage, MAI in each sub-carrier is firstly estimated using the symbol tentative decision from previous IC stage, and then is subtracted from $Z_{k, m}(i)$ in (2), and thus MAI suffered by each user is more or less cancelled. Perform decisions over the MAI-cancelled signals to obtain more reliable tentative values which are then input to the next IC stage. The whole IC procedure is demonstrated in Figure 1.

The estimated MAI for the $\mathrm{m}$-th sub-carrier is $\hat{I}_{k, m}^{(q)}(i)=\sum_{k^{\prime}=1, k^{\prime} \neq k}^{K} \frac{\sqrt{E_{c}}}{N} \hat{b}_{k^{\prime}}^{(q-1)}(i) \rho_{k^{\prime}, k}$, which is subtracted from $Z_{k, m}(i)$ to obtain

$$
Z_{k, m}^{(q)}(i)=\sqrt{E_{c}} \alpha_{k, m} b_{k}(i)+\Delta I_{k, m}^{(q)}(i)+n_{k, m}(i)
$$

where $\Delta \boldsymbol{J}_{k, m}^{(q)}=I_{k, m}(i)-\hat{I}_{k, m}^{(q)}(i)$. If the estimated MAI $\hat{I}_{k, m}^{(q)}(i)$ is more exact and closer to actual MAI $I_{k, m}(i), \Delta I_{k, m}^{(q)}$ is surely getting less, and system performance is expected to improve more dominantly.

The $\left\{Z_{k, m}^{(q)}(i), m=1,2, \cdots, M\right\}$ is subsequently input to the combiner, and we obtain by diversity combining

$$
Z_{k}^{(q)}(i)=\sum_{m=1}^{M} w_{k, m}^{(q)} Z_{k, m}^{(q)}(i)
$$

where $w_{k, m}^{(q)}$ is combining gain at this IC stage.

Similarly to (4), we have

$$
\hat{b}_{k}^{(q)}(i)=\mathrm{D}\left(Z_{k}^{(q)}(i)\right)
$$

which is subsequently sent to the next IC stage. have

At the Q-th IC stage, which is actually the last stage, we

$$
Z_{k}^{(Q)}(i)=\sum_{m=1}^{M} g_{k, m}^{(Q)} Z_{k, m}^{(Q)}(i)
$$

and

$$
\hat{b}_{k}(i)=\operatorname{HD}\left(Z_{k}^{(Q)}(i)\right)
$$

where $\mathrm{HD}(\cdot)$ is hard decision operation.

\section{DISCUSSION OF DECISION FUNCTIONS}

In general, decision function $\mathrm{D}(\cdot)$ mainly has two forms: hard decision and soft decision. 
After performing hard decision over $Z_{k}^{(q)}(i)$, we obtain

$$
\hat{b}_{k}^{(q)}(i)=\mathrm{D}\left(Z_{k}^{(q)}(i)\right)= \begin{cases}1, & Z_{k}^{(q)}(i)>0 \\ -1, & Z_{k}^{(q)}(i) \leq 0\end{cases}
$$

Otherwise if soft decision function [7] is used to $Z_{k}^{(q)}(i)$,

$$
\hat{b}_{k}^{(q)}(i)=\tanh \left(\frac{\mathrm{E}\left[Z_{k}^{(q)}(i) b_{k}(i)\right]}{\left(\sigma_{k}^{(q)}\right)^{2}} Z_{k}^{(q)}(i)\right)
$$

where $\left(\sigma_{k}^{(q)}\right)^{2}$ is the variance of $Z_{k}^{(q)}(i)$.

However, due to huge computation amount of nonlinear hyperbolic tangent function in (11), the application of soft decision method is restricted. Approximately, we can linearize nonlinear hyperbolic tangent function in (11) to reduce the computation comlexity as

$$
\hat{b}_{k}^{(q)}(i)=\left\{\begin{array}{c}
\frac{\mathrm{E}\left[Z_{k}^{(q)}(i) b_{k}(i)\right]}{\left(\sigma_{k}^{(q)}\right)^{2}} Z_{k}^{(q)}(i), \quad \frac{\mathrm{E}\left[Z_{k}^{(q)}(i) b_{k}(i)\right]}{\left(\sigma_{k}^{(q)}\right)^{2}} Z_{k}^{(q)} \leq 1 \\
\operatorname{sgn}\left(\frac{\mathrm{E}\left[Z_{k}^{(q)}(i) b_{k}(i)\right]}{\left(\sigma_{k}^{(q)}\right)^{2}} Z_{k}^{(q)}(i)\right)=\operatorname{sgn}\left(Z_{k}^{(q)}(i)\right), \quad \frac{\mathrm{E}\left[Z_{k}^{(q)}(i) b_{k}(i)\right]}{\left(\sigma_{k}^{(q)}\right)^{2}} Z_{k}^{(q)}>1
\end{array}\right.
$$

which is derived by using the approximation

$$
\tanh (x) \approx\left\{\begin{array}{c}
x, \quad|x| \leq 1) \\
\operatorname{sgn}(x), \quad|x|>1
\end{array} .\right.
$$

For convenience of description, We call the MC-PIC scheme corresponding to the decisions denoted by (10), (11) and (12) as Hard -MC-PIC, Soft -MC-PIC and Linear -MCPIC, respecticely.

\section{SIMULATION RESULTS}

In this section, we will make numerical analysis over the proposed MC-PIC scheme by the computer simulation. The main simulation parameters are set as follows: (1) the user number $\mathrm{K}=11$; (2) randomly generated spreading sequence with $\mathrm{N}=15$ for each user; and (3) sub-carrier number $\mathrm{M}=3$.

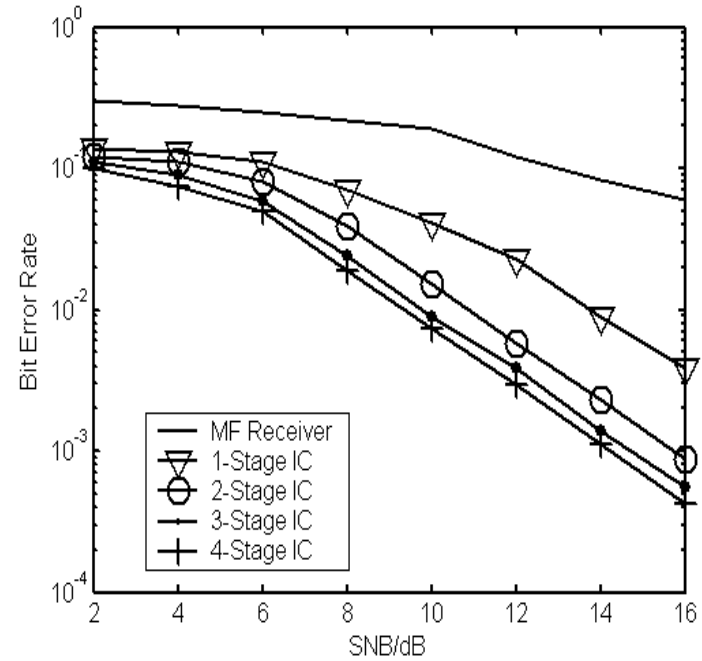

Figure 2. Performance of Linear-MC-PIC over Rayleigh fading channel.

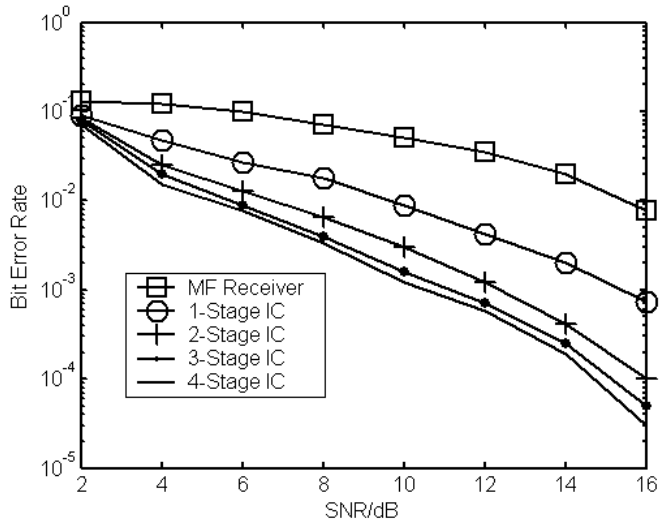

Figure 3. Performance of Linear-MC-PIC over AWGN channel.

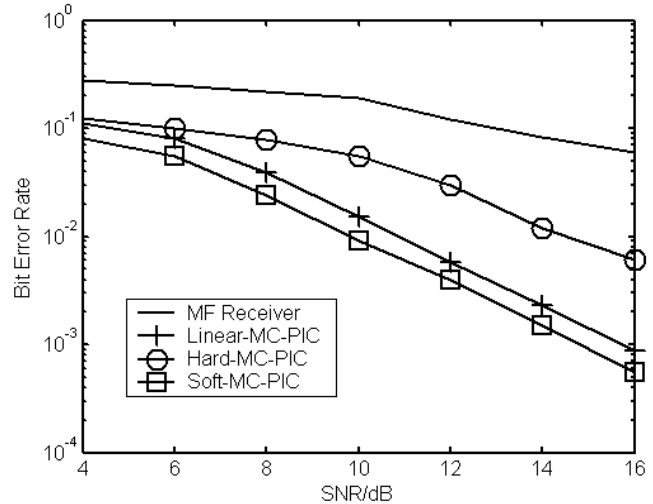

Figure 4. Performance comparison of Linear-MC-PIC, Hard-MC-PIC and Soft-MC-PIC in case of two IC stages.

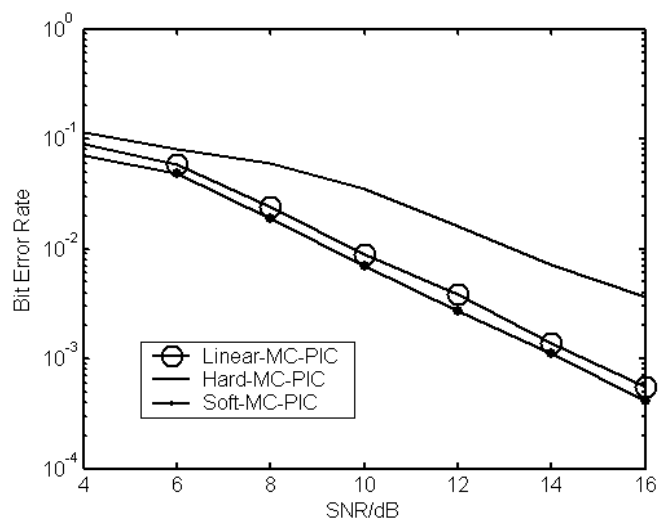

Figure 5. Performance comparison of Linear-MC-PIC, Hard-MC-PIC and Soft-MC-PIC in case of three IC stages.

Figure 2 and Figure 3 show the performance of LinearMC-PIC with different IC stages in two cases of Rayleigh fading channel and AWGN channel, respectively. Meanwhile, the bit error performance of the match-filtered (MF) receiver for multi-carrier DS-CDMA is also shown in both figures for comparison. It is clear from both figures that Linear-MC-PIC exihibits the performance superior to $\mathrm{MF}$ receiver, and the more IC stages, the better performance 
Linear-MC-PIC has, whether over Rayleigh fading channel or over AWGN channel. On the other hand, more IC stages doesn't bring more dominant performance improvement, and initial two IC stages can obtain a dominant performance gain, but more than two IC stages only improve the performance slightly. For example, in Figure 2, at signal-to-noise rate (SNR) of $12 \mathrm{~dB}$, the bit error rates of Linear-MC-PIC with one, two, three and four IC stages are, respectively, $2 \times 10-2$, $6 \times 10-3,4 \times 10-3$ and $3 \times 10-3$, while at the same SNR, the bit error rate of MF receiver is about 10-1, far higher than the bit error rates of Linear-MC-PIC.

Figure 4 and Figure 5 compare the bit error performance of Linear-MC-PIC, Hard-MC-PIC and Soft-MC-PIC with two IC stages and three IC stages, respectively. We can see from Figure 4 and Figure 5 that compared to Hard-MC-PIC, Linear-MC-PIC exhibits dominant performance advantage, whether with two IC stages or with three IC stages. On the other hand, Linear-MC-PIC is slightly interior to Soft-MCPIC in performance. For example, in Figure 4, at SNR of 14 $\mathrm{dB}$, the bit error rates of Hard-MC-PIC, Linear-MC-PIC and Soft-MC-PIC are about $1.2 \times 10-2,1.5 \times 10-3$ and $1.2 \times 10-3$, respectively. However, with the linearization of tangent function, Linear-MC-PIC has a lower computation complexity, relatively to Soft-MC-PIC.

\section{CONCLUSIONS}

In this paper, we explored a new parallel interference cancellation scheme for multi-carrier DS- CDMA. The detailed description and analysis over the proposed MC-PIC scheme were made, and the related discussion on decision functions was also performed, and a linear decision method is derived by modifying the soft decision function. Finally, the proposed MC-PIC scheme was simulated in performance. By the computer simulation, we drew conclusions, which are mainly as: (i) for the proposed PIC scheme, whether over Rayleigh fading channel or over additive white Gaussian moise (AWGN) channel, more IC stages bring larger performance gain, but with the further increase in the number of IC stages, the performance improves less dominantly; (ii) in case of the same IC stages, for the proposed PIC scheme, the linearization of soft decision function will bring the reduction of computation complexity with an ignorable performance loss. Therefore, from performance/complexity trade-off, MC-PIC scheme with linear decision is more suitable for practical application in future.

\section{REFERENCES}

[1] S.Kondo and L.B.Milstein, "Performance of multi-carrier DS CDMA systems," IEEE Trans. Commun., vol.44, pp.238 246, Feb.1996.

[2] Chouly, et al, " Orthogonal multicarrier technique applied to direct sequence spread spectrum CDMA systems," In Proc. GLOBECOM, Houston, TX, pp.1723 1728, Nov.1993.

[3] S.Moshavi, "Multi-user detection for DS-CDMA communications," IEEE Commun. Mag., vol. 34, pp.124 137, Oct.1996.

[4] A.L.C.Hui and K.B.Letaief, "Successive interference cancellation for multiuser asynchronous DS/CDMA detectors in multipath fading links," IEEE Trans. Commun., vol.46, pp.384 391, Mar.1998.

[5] L.Fang and L.B.Milstein, "Successive inter- ference cancellation in multicarrier DS/ CDMA," IEEE Trans. Commun., vol.48, pp. 1530 1540, Sept. 2000.

[6] G.X.Xu, "Turbo IC/decoding for multi-carrier DS-CDMA," in Proc.of ICCC2013, Wuhan, pp.74-77, Nov.2013.

[7] L. C. Gan and G.X.Xu, "Turbo interference cancellation/decoding for cnvolutionally coded multi-carrier DS-CDMA," Chinese Journal of Electronics, vol.14,pp.309-313, April 2005. 